\title{
LA FEDERACIÓN LATINOAMERICANA DE SEMIÓTICA. ¿EXISTEN LOS SEMIÓLOGOS LATINOAMERICANOS?
}

\section{Lucrecia Escudero}

Vicepresidenta de la Federación Latinoamericana de Semiótica

Como en un cuento de Julio Cortázar, los primeros semiólogos latinoamericanos se encontraron en París. No porque no hubiera semiólogos en Latinoamérica, sino simplemente porque la condición de «latinoamericanidad» se desarrolla, preferentemente, cuando uno está en el extranjero. Se encontraban en el mítico 10 de la calle de Monsieur le Prince, sede del Grupo de Investigaciones Semio-linguísticas de la Escuela de Altos Estudios en Ciencias Sociales y donde el no menos legendario Algirdas Julien Greimas dictaba su seminario de Semántica General en el año académico de 1984.

Muchos latinoamericanos pasaban por París y sus universidades, pero el seminario de Greimas fue un foco de formación y de pasaje obligado de aquéllos que querían formarse en semiótica. Al principio asistían juntos a las clases, luego formaron un «Grupo de discusión» preocupados por problemas de metodología y rápidamente se dieron cuenta que les unía una misma obsesión: todos analizaban 
corpus latinoamericanos. La mirada trasversal de la semiótica para incorporar al intelectual periférico a un debate universal. La fidelidad a una postura que encontraremos en la base de la identidad latinoamericana: lo que se analiza, para lo que se estudia, es para comprender los problemas que pone el sentido «de allá». La fascinación, siempre renovada, por la lectura y la construcción metodológica de objetos latinoamericanos, los mitos, las narraciones, la arquitectura, los medios, la literatura, la política. Por otra parte, estos estudiantes compartían todos una demanda parecida: el creciente desarrollo de las carreras sociales en América Latina postulaba una formación semiológica. A diferencia del desarrollo académico europeo, mucho más rígido y anclado en tradiciones y trayectorias epistemológicas que son muy difícilmente innovables, en la América Latina de los años ochenta, se asistió a un verdadero boom de carreras de comunicación social que requerían de la semiótica para leer a los medios, de maestrías en estudios literarios que cruzaban la antropología y la sociología, o formaciones de arquitectura donde la semiótica era una de las disciplinas claves para desentrañar la noción de lenguaje.

Porque de eso se trataba: del sueño de Saussure de estudiar la vida de los signos en el seno de la vida social, de la utopía de Barthes para quien la semiología sería la ciencia de la connotación, de la profecía de Eco para quien la mirada sobre los medios no podía ser de ningún modo apocalíptica. Sueños europeos floreciendo en América, como siempre ha sido desde el sueño de la conquista: es la mirada del otro el que nos funda. Sólo que aquí se producía una inversión los: latinoamericanos eran mayoría en la calle de Monsieur le Prince y -como me lo confesó muchos años después Graciela Latella- en realidad también descubrieron su identidad para hacerse notar frente a los franceses, atacados siempre por esa pasión de la indiferencia, prima hermana del esnobismo inglés.

Con unidad de corpus - la problemática traída de sus propios países--, la unidad de lengua —el español—, la unidad de acción -el seminario-, el grupo se constituyó como Grupo Latinoamericano de Semiótica al año siguiente. Pero como estaban en París y los franceses son formales, Liddy Palomares de Mendoza, Teresa Espar e Iván Ávila Belloso de Venezuela, María Cuculiza del Perú, el antropólogo mexicano Roberto Flores, los argentinos Graciela Latella y Donaldo Dib, que trabajaba en arquitectura, se acogieron a la ley francesa de 1901 de asociaciones sin fines de lucro y formalizaron el Grupo en el año 1985. 


\section{EL PRIMER CONGRESO LATINOAMERICANO DE SEMIÓTICA (1985)}

Durante ese mismo año de gracia y cruzando el océano, José Pascual Buxó presidía la Asociación Mexicana de Semiótica y dirigía desde 1978 el famoso seminario de Poética de la Facultad de Filosofía y Letras de la Universidad Nacional Autónoma de México y en la ciudad de Puebla Adrián Gimate Welsh había creado la maestría en Ciencias del Lenguaje con una fuerte matriz semiológica y era a su vez el vicepresidente de la Asociación mexicana. Yo había conocido a Gimate Welsh en el III Congreso de la Asociación Internacional de Semiótica en Palermo, donde muchos votamos a favor de su candidatura como representante de México.

Trabajando ya con ellos en México, tuvieron la idea de invitar a Umberto Eco a dictar una serie de conferencias en la UNAM. Eco llegó a mediados del 85 y la enorme multitud que se apelotonó en la entrada del paraninfo de la UNAM, bajo una lluvia torrencial como suele llover sólo en América Latina, confirmó no sólo la celebridad mundial del semiólogo italiano sino el enorme interés que suscitaba la semiótica en estudiantes de todas las carreras. Y así surgió el Primer Congreso Latinoamericano de Estudios Semióticos, que se desarrolló en la UNAM y en la Universidad Autónoma de Puebla del 9 al 13 de setiembre de ese año.

Una mirada al programa de actividades muestra, luego de las palabras de inauguración a cargo de José Pascual Buxó, la conferencia inaugural del congreso a cargo de Paolo Fabbri, de la universidad de Bologna, quien en su primer viaje a América Latina eligió hablar de «La semiótica y su mito», presagiando ya el boom de los estudios semióticos latinoamericanos. Los principales investigadores e intelectuales mexicanos interesados en estos estudios estaban presentes, como Mauricio Beuchot, Mario Valdés, que trabajaba en la universidad de Toronto, Antonio Alcalá Alba, el arquitecto Antonio Toca Fernández, que había escrito un importante libro sobre el desarrollo de la semiótica de la arquitectura en América Latina o Renato Prada Oropeza, de la universidad Veracruzana. Las intersecciones estaban marcadas: los ejes de discusión del congreso incluyeron una mesa sobre semiótica, epistemología y hermenéutica con la presencia de Walter Mignolo, de la universidad de Michigan, Carlos Pereda y Raúl Quesada de la UNAM. Estudiantes ya formados en sus años superiores como 
Esther Cohen -que había pasado por la universidad de Bologna, siendo alumna de Fabbri - o Luisa Ruiz Moreno, que estaba haciendo su tesis con Pascual Buxó, presentaron ponencias, ante un público universitario de aproximadamente ochocientos inscritos.

La mesa de semiótica y literatura incluía conferencias sobre la influencia de Iury Lotman y en la de semiótica de la cultura, moderada por el profesor argentino Noé Jitrik, que dirigía el Centro de Análisis del Discurso de la UNAM, se discutían las identidades masculina/femenina y una aproximación a la lectura femenina que marcaron con notable antelación los estudios culturalistas sobre la mujer que se desarrollarían en la década de los noventa. Peirce estaba presente en la reflexión de Raimundo Mier. La mesa de semiótica y artes visuales ejemplificó una de las obsesiones claves de la semiótica latinoamericana, el espacio arquitectónico, con ponencias sobre la teoría de la delimitación espacial del semiólogo y arquitecto argentino Cesar Iannello (Escudero Chauvel), la arquitectura como praxis significante (Gordillo) o el ornamento como figura retórica (Fornari). Wladimir Krysinski de la universidad de Montreal trabajó los estudios de Bajtín y Eric Landowsky de la universidad de París, analizó los discursos del poder ${ }^{1}$ en la mesa de semiótica y ciencias sociales moderada por Gilberto Giménez, uno de los analistas más lúcidos del discurso político mexicano. Adrián Gimate Welsh presentó su importante investigación sobre uno de los temas claves de la producción de sentido de los discursos hegemónicos como eran los programas de acción del PRI.

Este primer encuentro universitario, donde los semiólogos latinoamericanos nos nombrábamos a nosotros mismos como tales, trazó en cierta medida las líneas de una posición epistemológica: el pluralismo de las voces y de las temáticas, de horizontes muy diferentes, el encuentro entre europeos y latinoamericanos, la mirada semiótica cruza otras prácticas sociales para trabajar allí donde opera la significación. El profesor Jerzy Pelc presidente de la Asociación Internacional de Semiótica pronunció las palabras de clausura junto a un emocionado Adrián Gimate Welsh. Cinco días después de terminado el congreso, y mientras todos festejábamos el éxito del mismo y despedíamos a los huéspedes en los aeropuertos, el fatal terremoto de ciudad de México arrasó el centro histórico de una de las ciudades más emblemáticas

\footnotetext{
${ }^{1}$ Escudero Chauvel, L. [19921 «Appocalitto e integrato» en Semiótica: Storia, Teoria, Interpretazione. Saggi Intorno a Umberto Eco. Milano: Bompiani. Landowski, E. [1986] «Pour une approche semionarrative du droit. En Actes Semiotiques, vol. VIII, número 71 .
} 
de Latinoamérica. Recuerdo perfectamente que Paolo Fabbri, a sólo doce horas antes de producirse el cataclismo, me preguntó mientras nos despedíamos: «QQué tiempo raro que hace! ¿En qué estación del año estamos?».

\section{EL SEMINARIO INTERNACIONAL DE LA UNIVERSIDAD NACIONAL DE ROSARIO (1986)}

Y en ese mismo año de 1985 volví a la universidad de Rosario, en la Argentina de la recientemente inaugurada democracia del presidente Raúl Alfonsín, luego de los años más sombríos y crueles de la dictadura militar. Las cárceles se habían abierto, el juicio a los culpables de una de las masacres más cruentas de la historia contemporánea de nuestra América se iniciaba, así como en todas las facultades y universidades de la República se inauguraban placas recordatorias de los profesores y alumnos desaparecidos. La Escuela de Comunicación Social, que funcionaba en la Facultad de Ciencias Políticas de la Universidad Nacional de Rosario tenía el sombrío antecedente que sus locales habían funcionado al lado de la sede del comando del IIdo. cuerpo del Ejército que había sido un conocido centro de tortura. Y además estaba la memoria de los muertos. La universidad estaba desmantelada, los fondos de las bibliotecas expurgados de los textos prohibidos, el estructuralismo, la semiótica, el psicoanálisis y la teoría matemática de los conjuntos habían sido abolidos de cualquier formación institucional por subversivos. Es conocida la anécdota de la quema del Rojo y negro de Stendhal por obvias connotaciones que Barthes hubiera adorado si no hubiese marcado como una metáfora de fuego a casi una década de argentinos.

Durante el final de ese año académico con Susana Frutos, directora de la Escuela y que había regresado a su vez de Colombia, preparamos un programa de terapia intensiva. Teníamos que encontrar el modo por el cual los estudiantes y graduados de comunicación conocieran los nuevos paradigmas de investigación en el sector y dotar a los ya egresados, que no habían tenido acceso a ningún nivel de perfeccionamiento de postgrado, de un instrumental teórico sólido para elevar el nivel de la didáctica de grado. En un primer momento, hicimos una encuesta general entre los estudiantes y graduados en el área de ciencias sociales y letras para establecer un orden de prioridades y de inte- 
reses colectivos. Así surgió una primera lista de temas que abarcaban desde las teorías de la comunicación, al análisis del discurso y la semiótica aplicada a los medios, los diferentes objetos de la cultura de masas y su forma de abordaje metodológico.

Se nos abría un panorama interdisciplinario rico y variado y entonces nuestra elección organizativa se planteó con claridad: crear un espacio transdisciplinario de discusión y enfoque de estas problemáticas invitando en forma abierta a profesores especialistas de diferentes universidades latinoamericanas, canadienses y europeas a dictar un seminario intensivo de una semana de duración y compartiendo con otras facultades del interior del país y de Buenos Aires la asistencia de alumnos, graduados y profesores. La Asociación Argentina de Semiótica, que había sido una de las primeras en fundarse en 1973, bajo la iniciativa de Eliseo Verón, Alicia Páez, Óscar Steimberg y Óscar Traversa, se estaba reestructurando, con la actividad del arquitecto Claudio Guerri y de la profesora Rosa María Ravera, quien representó a la Argentina desde la creación de la Asociación Internacional de Semiótica.

En la mesa del comedor de mi abuela, Susana Frutos, Olga Corna y yo -que ya habíamos sobrevivido juntas a muchas guerras- escribimos la primera lista de semiólogos y estudiosos sociales invitados. Lo de «invitados» era una temeridad: la universidad no tenía un peso y había que arreglárselas con «una lata y un palito» como decía Olga. Pero la respuesta de la comunidad latinoamericana e internacional apoyando al programa fue inmediata y conmovedora. Todos nos contestaron y algunos, que no habían sido incluidos en la lista original, se postularon para trabajar. Inmediatamente comprendimos que había una gran cantidad de profesores, de excelente nivel académico y seguramente excelente salario, dispuestos a donar una semana de su tiempo en forma gratuita a una universidad de provincia, contribuyendo a un debate sobre la re-estructuración de los currícula, los contenidos de las cátedras de semiótica y las referencias bibliográficas de base.

Los invitados fueron llegando. Eliseo Verón ${ }^{2}$ inauguró el seminario el 10 de abril de 1986 como un homenaje a uno de los teóricos que más han marcado el desarrollo de la semiótica latinoamericana, con una conferencia sobre la postmodernidad y el fin de los funcionalismos.

2 Verón, E. [1985]: «Post-modernité et théories du langage: la fin des fonctionnalismes» en: Georges Orwell et L'Univers de L'Information. París: Centre Georges Pompidou. 
Noé Jitrik, que todavía vivía en México cuando contestó la invitación, se contó entre los primeros en llegar con su solidaridad, sus propuestas de trabajo y sus buenas ideas. Paolo Fabbri desembarcó y habló durante cinco días seguidos en un seminario memorable sobre la semiotización de las pasiones. Hubo que pedir el principal teatro de la ciudad prestado, pues los alumnos eran infinitos, y por una vez la estructura arquitectónica acompañó sus palabras, mientras escuchábamos a María Callas en el final de Norma para ejemplificar su teoría de las pasiones. Cuando llegó el semiólogo canadiense Paul Buissac fuimos todos al circo y allí analizó la evolución de la semiótica del sistema del circo europeo y su transformación vernácula, en esa escena de arena de la periferia de un bamo rosarino y ante la mirada asombrada de payasos y domadores. Christian Metz arrastró a los alumnos al cine y releyó su célebre Le cinema: langue ou langage? de 1964 con la mirada epigonal del que iba a ser tal vez su último viaje a América Latina ${ }^{3}$.

Los italianos no se hicieron esperar, y llegaron Lamberto Pignottti analizando el mensaje publicitario, y Roberto Grandi, Antonio Cascino y Piero Dorfles discutiendo los nuevos enfoques del marketing político, la legislación italiana en medios de comunicación y la posición teórico-ideológica de los investigadores de la comunicación en sociedades post-industriales. Pier Aager Brandt y Herman Parret regresaron luego varias veces a la Argentina, a partir de ese primer contacto con los estudiantes del seminario ${ }^{4}$. Finalmente Adrián Gimate Welsh y José Pascual Buxó llegaron hasta Rosario con la amistad, inteligencia y generosidad de siempre, para trabajar con nosotros el análisis de las ideologías. Pasaron más de mil trescientos estudiantes por los seminarios ${ }^{5}$, algunos compartidos con la Escuela de Graduados de la Facultad de Humanidades, que dirigía por entonces el crítico y semiólogo de la literatura Nicolás Rosa. Asistieron profesores y estudiantes de las universidades de Buenos Aires, La Plata, Entre Ríos, Misiones, Córdoba, Río Cuarto y Cuyo. El archivo y fichero de Seminario sirvió como base para realizar los congresos de la Asociación Argentina de Semiótica, pero sobre todo un proyecto ambicioso en Seuil.

3 Metz, C. [1964]: «Le cinema: langue ou langage?», en Communication, 4. París:

${ }_{4}$ Parret, H. (1986): Les passions. Essai sur la mise en discours de la subjectivité. Bruxelles: Pierre Mardaga. El texto «Lettre sur les passions» está firmado en la ciudad de Córdoba el 19 de octubre de 1987.

5 Todos los seminarios están publicados en Escudero, L.; Corna, Ó. (eds.) [1992]: Seminario Internacional. Comunicación, discursos, semióticas. Rosario: UNR Editora. 
común con otras universidades latinoamericanas: el Segundo Congreso Internacional Latinoamericano de Semiótica.

Escribiendo estos nombres, recordando las discusiones, las críticas y las defensas al Seminario, el tiempo transcurrido, los que pasaron y los que volvieron, en síntesis ese think tank que fue el Seminario en esos años, creo que no nos equivocamos en nuestra intuición inicial: la universidad es una aventura de la gente, las instituciones se llenan o se vacían y conocimiento y experiencia humana son dos de las múltiples caras que tiene la interacción de la producción de saber en ciertos escenarios sociales. Las otras caras posiblemente sean la inversión sostenida y la libertad. Pero el diseño se iba completando: la semiótica latinoamericana se desarrolla estrechamente vinculada con el diálogo europeo y la formación de las cátedras académicas. Una de las especificidades es precisamente este anclaje en lo institucional que produce la demanda de formación de especialistas y el dictado de las cátedras en currículas integradas. La otra vertiente es, sin duda, el diálogo con los europeos. A propósito, ¿en qué andaban los latinoamericanos anclados en París?

\section{EL PRIMER COLOQUIO DE SEMIÓTICA LATINOAMERICANA}

Al inicio del siguiente año académico, el Grupo Latinoamericano de Semiótica produjo un golpe de visibilidad. En enero de 1986 abríamos un sobre con una invitación a participar en el «Primer Coloquio Internacional Latinoamericano de Semiótica» cuyo título «Identidad e Interacción» era de por sí todo un programa narrativo. El Coloquio se desarrolló en el mes de enero casi inmediatamente después del encuentro de México y a causa de la eterna división de los hemisferios, en plenas vacaciones argentinas inmediatamente antes del lanzamiento del Seminario. El Coloquio fue una especie de bisagra que nos permitió re-encontrarnos y, por la casi simultaneidad de los eventos, contribuyó a legitimar la identidad de una «semiótica latinoamericana» que se iba articulando a fuerza de reflexión, voluntad de encontrarse y de discutir, curiosidad por la práctica del otro. El Coloquio, que se desarrolló en la Maison de la Amerique Latine en Paris, con los auspicios de la UNESCO, tuvo unos cuarenta ponencias y una gran respuesta de público, obviamente estudiantes latinoamericanos. Pero 
lo interesante de esta experiencia fue precisamente el entrecruzamiento Europa/América.

Iván Ávila Belloso era el presidente del comité organizador, teniendo como alfiles a Graciela Latella, Roberto Flores, María Cuculiza y Liddy de Mendoza en las filas del secretariado. Donaldo Dib se firmaba «arquitecto» y era el encargado de la tesorería. Con las palabras de Algirdas J.Greimas se inauguró el Coloquio, seguido de una serie de plenarias entre las que destacaba la de Teresa Espar, de la universidad de los Andes, quien en esta primera conferencia resumía en el título una de las interacciones más fecundas: «Semiótica, literatura y mestizaje». Yo coordiné junto a Donaldo Dib el taller de semióticas no verbales donde se discutieron los problemas de la constitución del sujeto, entre identidad y sincretismo, según la acertada expresión de Ávila Belloso. Roberto Flores coordinó el taller de etno-semiótica con la presencia de Gimate Welsh en un análisis del discurso nacionalista oficial como expresión de identidad de la sociedad mexicana, y Enrique Ballón Aguirre, de la Universidad de San Marcos, en Perú, trabajó sobre la mítica de los indios huitoto. En el taller de semiótica literaria, coordinado por Graciela Latella, se trató sobre los grandes nombres de la literatura latinoamericana, lo real maravilloso de Alejo Carpentier (Liddy Mendoza), las transformaciones borgeanas (Latella), la identidad en la narrativa de García Márquez (Teresa Mozejko) ${ }^{6}$ y la obra de Clarice Lispector (Elba Bohorquez). El aspecto de la interacción metodológica con un corpus latinoamericano apareció claramente en los tres talleres de etnosociosemiótica coordinados por Mana Cuculiza, Roberto Flores y Hermis Campodonico, con los trabajos de Roberto Flores (la Historia de Indias de Pedro Durán), Hermis Campodonico y Rafael Resendiz (lo radiofónico), Gabriel Hernández (la revolución mexicana), Hilda Díaz (el mito de Coyolxauqui) y Luisa Ruiz Moreno (el espacio sincrético en América Latina).

Me parece imprescindible señalar en este elenco de nombres la intervención de François Rastier y Per Aager Brandt con un análisis de la poética de Jorge Luis Borges; de Iván Darrault sobre la identidad patológica del sujeto; de Manar Hammad con su estudio de la casa del te japonesa ${ }^{7}$ y de Paolo Fabbri problematizando uno de los leit-motifs

${ }^{6}$ Teresa Motzeiko de Costa ya había publicado en Actes Semiotiques su trabajo «Enoncé et enonciation chez Octavio Paz», 1 (984), vol. VI.

7 Hammad, M. [1987] «La architecture du thé». En Actes Semiotiques, números 8485 , vol IX. 
de sus investigaciones: la identidad del doble agente secreto ${ }^{8}$. Dos nuevos personajes aparecerán en escena: Norma Tasca, estrechamente ligada al grupo greimasiano y fundadora de la Asociación Portuguesa de Semiótica, que participó en la mesa de semióticas literarias y el colombiano Armando Silva, de la universidad de Bogotá, que trabajó el tema del graffiti. Visto en perspectiva, el coloquio de París permitió nuclear a casi todos los investigadores que luego constituirían la Federación Latinoamericana de Semiótica.

Pero también marca un momento de la discusión en el interior de la escuela de semiótica greimasiana, donde sus principales exponentes participaban intensamente en nuestros encuentros y publicaban a posteriori el resultado de sus conferencias dictadas en América Latina. ¿Habremos sido los latinoamericanos la caja de resonancia de los debates europeos? El coloquio marcó también un deslizamiento de la conjunción del «V» (semiótica y filosofía, semiótica y arquitectura) que caracterizó a la primera reunión mexicana, al de un campo problemático establecido a partir de la semiótica: etno-semiótica, sociosemiótica, semiótica literaria. Se pasó de la intersección de dos universos que podrían complementarse (desde la semiótica hacia la filosofía o hacia la sociología) a un debate intra-semiótico de establecimiento de territorios.

Lo cual vuelve aún más vigente la distinción que ya hacía Umberto Eco en 1984 -y que re-elabora en cierto modo la del Tratado de Semiótica General de 1975 entre «campo» y «disciplina»-entre una Semiótica General, de naturaleza filosófica porque coloca las categorías a partir de las cuales pensar los sistemas de significación y poder compararlos y las semióticas aplicadas o específicas que postularan sus propias categorías internas —una «gramática de un particular sistema de signos» dirá Eco- en la diversidad de los soportes de análisis elegidos. Los dos pioneros encuentros latinoamericanos señalan paradójicamente este movimiento de lo general a lo particular, pero a su vez el de una ciencia en diálogo con otras disciplinas. Si lo latino se había fundado siempre en la variedad de los corpus bajo el imperio de una mirada semiótica, ¿cómo pasar de lo particular a lo general? $\mathrm{O}$

${ }^{8}$ Esta célebre conferencia fue reproducida como artículo en versión francesa [1988]: «Todos somos agentes dobles», en Le Genre Humain. La Trahison. París: Seuil; en versión española [1988] en Revista de Occidente, $\mathrm{n}^{\circ} 85$. Madrid. Finalmente incluye la selección de textos en español en Fabbri, pág. 1, 1995. Tácticas de los signos. Barcelona: Gedisa. Eco, U [1984]. Semiótica e filosofia del linguggio. Milano: Einaudi, págs. XI-XII. 
dicho de otro modo, es posible una semiótica latinoamericana que combine la fidelidad al regionalismo de las temáticas y de los países con una reflexión sobre las leyes generales que colocan las categorías del sentido?

\section{EL SEGUNDO CONGRESO LATINOAMERICANO DE SEMIÓTICA (1987)}

El Segundo Congreso Internacional Latinoamericano de Semiótica tuvo lugar en la Universidad Nacional de Rosario a principios del mes de octubre de 1987.

Como una continuación de los anteriores y con la experiencia del Seminario, la Escuela de Comunicación Social y la Facultad de Ciencias Políticas pusieron el pie de imprenta. Vinieron ponentes de más de setenta universidades latinoamericanas donde se enseñaba orgánicamente semiótica. Para paliar la ausencia de los investigadores brasileros en los anteriores encuentros, decidimos constituir un comité ejecutivo con miembros por países, a fin de asegurar una presencia integral del continente. El comité, presidido por José Pascual Buxó, contó con Adrián Gimate Welsh por México. Monica Rector, de la Universidad Federal de Río de Janeiro y una de las fundadoras de la dinámica Asociación Brasilera de Semiótica en 1979, María Lucía Santaella Braga, que dirigía el programa de postgrado en Semiótica -de inspiración peirciana - en la Pontificia Universidad Católica de San Pablo, Diana Luz Pessoa de Barros que coordinaba el grupo de semiótica brasileña que trabajaba en la línea greimasiana de la Universidad del Estado de San Pablo y Eduardo Peñuela Cañizal de la misma universidad completaban la representación brasilera. A los conocidos Iván Ávila Belloso y Teresa Espar que venían en representación de Venezuela, se sumaban Graciela Latella del Grupo Latinoamericano de Semiótica de París e incluimos por primera vez a Armando Silva por Colombia, Luis Torres Acuña por Chile —quien luego sería reemplazado por Rafael del Vilar-, Desiderio Blanco por Perú y Lisa Block de Behar por Uruguay.

Nos dimos un objetivo en común: trazar un mapa de los estudios de semiótica en América Latina y ver cómo se podía organizar un intercambio más sostenido entre nuestros colegas. El encuentro de Rosario 
tuvo en realidad una preocupación práctica: las cátedras de semiótica se multiplicaban en nuestras universidades, teníamos que hacer un balance de las temáticas desarrolladas, de los profesores, de los programas, de las áreas de interés. Por otra parte, la pregunta del coloquio de París seguía en pie y por eso el congreso de 1987 se llamó «De la práctica a la teoría». La presidencia de honor fue ofrecida simultáneamente a Umberto Eco y Algirdas J.Greimas en reconocimiento a la influencia fundamental en la reflexión latinoamericana.

Nuevamente Eliseo Verón llegó en nuestro auxilio, inaugurando el congreso con una conferencia sobre semiótica y democracia donde se preguntaba qué significaba trabajar en semiótica en los años ochenta, en los diferentes contextos latinoamericanos y europeos y con qué instrumentos conceptuales se trabajaba. En la perspectiva de Verón ${ }^{9}$ era posible esbozar un primer diagnóstico: la semiótica latinoamericana nacía en el contexto de las ciencias sociales durante el final de la década de los años sesenta, dominada por las grandes síntesis globales - la utopía de los relatos totalizantes- para caer una década después en la crisis de las democracias industriales con su visión parcializada de los procesos de sentido. La recesión global de los años ochenta y las «grandes profecías» —optimistas o no- ilustraban la emergencia de una mediación social y la producción global de la industria de la cultura, lo que influía en América Latina en el desenvolvimiento de una semiótica aplicada y no una Semiótica General. Segun Verón, el primer efecto de este parcelamiento fue académico: del otro lado del Atlántico la universidad tradicional reconoció tardíamente a la semiótica como disciplina académica, mientras que en territorio latinoamericano ésta apareció fuertemente ligada a las prácticas interpretativas de las semióticas aplicadas, de las consultorías y del trabajo de los semiólogos en cuanto profesionales integrados.

La estructura del congreso mostró su preocupación por la integración latinoamericana. Dividido en tres jornadas de trabajo, la primera estuvo presidida por Adrián Gimate Welsh, dedicada al estado de la investigación semiótica en América Latina y exploró el desarrollo en cada país en particular. La segunda jornada, presidida por el crítico literario y semiólogo de la literatura Nicolás Rosa (Argentina), estuvo dedicada al análisis de la obra de Borges con comunicaciones de Wladimir Krisinsky, Iván Almeida y Per Aage Brandt. La tercera jornada,

${ }^{9}$ Re-elaborado en Verón, E. [1993]: «Mediatización, comunicación política y mutaciones de la democracia». México: Monterrey (mimeo). 
coordinada por María Lucia Santaella Braga (Brasil), llevaba por título «Sobre los paradigmas teóricos en la constitución de los estudios semióticos latinoamericanos» y presentaba las relaciones de diferentes disciplinas en la constitución de la(s) teoría(s) semiótica(s). José Romera Castillo- que entonces era el Secretario general de la Asociación Española de Semiótica - se refirió a la semiótica de los discursos y a las tres similaridades de la emergencia del campo teórico en España y América Latina: entusiasmo, incremento de los estudios con la llegada de las nuevas democracias y «colonización» teórica. Noé Jitrik trabajó el espacio discursivo, así como Enrique Ballón Aguirre y José Enrique Finol, de la universidad del Zulia en Venezuela, abordaron la etnosemiótica.

Hubo ocho mesas redondas dedicadas a las Figuras del Discurso, a la Comunicación e Interacción, a la Persuasión y manipulación, para marcar el cruzamiento de ejes de investigación y problemáticas típicas de la semiótica de los años ochenta. La mesa Cultura y Transición a la democracia fue una concesión al clima político que se vivía en América Latina luego de la caída de las grandes dictaduras militares argentina, chilena y uruguaya y porque en realidad los intelectuales latinoamericanos se articulan siempre en función de una reflexión política. La mesa «Espacios» reflejó lo que siempre he considerado una obsesión latente de la semiótica de la arquitectura ${ }^{10}$.

Para finalizar la semana, Paul Buissac, Per Aager Brandt, Iván Darrault y Herman Parret dictaron seminarios. Entre los nuevos se contaban José Romera Castillo, Patrick Imbert, Iván Almeida y José Enrique Finol, que dictó un seminario sobre el mito guajiro. Lo sorprendente de la riqueza y variedad de actividades que propusimos a los ochocientos congresistas, fueron las muestras de vídeo-arte organizada por Eduardo Peñuela Cañizal de la Escuela de Comunicación y Artes de la Universidad Estatal de San Pablo, la muestra de vídeos del Instituto Superior de Arte y Ciencias Sociales de Chile, la muestra experimental de vídeo-arte de Alaeteu del Perú y el show de vídeosclip coordinado por el colombiano Armando Silva. La UNAM y la Universidad Autónoma de Puebla estuvieron presentes organizando la Feria del Libro, donde nos encontramos con todas las publicaciones de semiótica de América Latina; mientras que la Facultad de Arquitectu-

${ }^{10}$ Escudero-Chauvel, L. [1988]: «The Second International Congres in Semiotics», en Sebeok, T. \& Urnrike - Sebeok, J. (eds): The Semiotic Web. Berlín-New York-Amsterdam: Mouton de Gruyter. 
ra y Urbanismo de la Universidad de Buenos Aires presentaba una muestra de investigaciones sobre el espacio de la cátedra Sistemas Visuales que coordinaba Claudio Guerri.

\section{LA CREACIÓN DE LA FEDERACIÓN LATINOAMERICANA DE SEMIÓTICA}

Mientras escuchábamos a un cantante de tangos peinado con gomina y comíamos empanadas criollas, el grupo organizador tuvo una misma pregunta: ¿cómo podía ser que intelectuales que se habían frecuentado poco, atravesados por las distancias y los desfallecientes correos latinoamericanos, que algunos inclusive era la primera vez que se encontraban, se habían podido conjugar tan armónicamente para producir un espectáculo colectivo que duró siete días sin fisuras ni desfallecimientos? Esa noche, volviendo al Hotel Italia, un edificio italianizante de la belle époque rosarina y donde paraba Borges cuando pasaba por Rosario - y contaba la leyenda, había pernoctado Gardel- esa noche, decidimos fundar la Federación Latinoamericana de Semiótica.

«Nosotros, los abajo firmantes, responsables de diferentes asociaciones y grupos de investigación de Semiótica, constituimos hoy, 7 de octubre de 1987, en la ciudad de Rosario, Argentina, la Federación Latinoamericana de Semiótica», decía el preámbulo de nuestra declaración de principios y de identidad. El Acta de Rosario, escrita a mano, salvando las diferencias - el acta de creación de la Asociación Internacional de Semiótica firmada por Roland Barthes y Julia Kristeva, y de la que Claudio Guerri guardaba una fotocopia fetiche, representaba un programa de trabajo y de compromiso importante. Vale la pena transcribirla porque en ella se mezcla el lenguaje formal de un grupo que empezaba a reconocerse legítimamente, con las utopías del desarrollo sostenido de una ciencia:

«En la ciudad de Rosario, provincia de Santa Fe, República Argentina, a los siete días del mes de octubre de mil novecientos ochenta y siete, reunidos en el Centro Cultural Bernardino Rivadavia, los abajo firmantes, en su carácter de integrantes y/o representantes de las Asociaciones de Semiótica de México, Venezuela, Brasil, Argentina, Uruguay, Perú, Colombia y del Comité Ejecutivo del Segundo Congreso Internacional Latinoamericano de Semiótica, deciden fundar la Fede- 
ración Latinoamericana de Semiótica, que tendrá su domicilio rotativo en las distintas Asociaciones de los países integrantes y cuyo objeto será el desarrollo de la investigación semiótica latinoamericana, la creación de una publicación como órgano, el establecimiento de nexos con instituciones nacionales e internacionales que apoyen la difusión de los estudios semióticos y el impulso de los mismos en las universidades latinoamericanas. A los fines del cumplimiento de dicho objetivo social se designa desde ahora como Comisión Provisoria al Comité Ejecutivo del Segundo Congreso Internacional Latinoamericano de Semiótica, la duración de cuyo mandato será de no más de dos años. Tendrá como misión principal la redacción de los estatutos de la Federación teniendo en cuenta las sugerencias recogidas en las diferentes Asociaciones nacionales y regionales, que luego serán remitidos a la aprobación de la Asamblea que deberá reunirse a tal efecto, dentro del mismo término fijado para la duración del mandato de la Comisión Provisoria y la organización del Tercer Congreso Internacional Latinoamericano de Semiótica. En prueba de conformidad, los otorgantes firman la presente acta en el lugar y fecha indicado up supra, incluyendo como miembros de la Federación a la Asociación Española de Semiótica, a la Asociación Canadiense de Semiótica y a la Asociación Portuguesa de Semiótica, e invitan a suscribir la presente Acta a los integrantes presentes de todas las Asociaciones Latinoamericanas de Semiótica y demás instituciones». Y firmaron María Lucía Santaella Braga, Aluizio Ramos Trinta, Eduardo Peñuela, Diana Luz Pessoa de Barros, Elisabeth Bastos Duarte, Cidmar Teodoro País, Nieves Ferreira Pintol, José Luis Fiorim, por Brasil; Óscar Quezada Macchiavello, por Perú; Carlos Pellegrino, por Uruguay; Iván Ávila Belloso y Enrique Finol, por Venezuela; Rosa María Ravera, Claudio Guerri, Olga Corna y yo, por Argentina; Luis Torres Acuña y Rafael del Villar, por Chile; Armando Silva por Colombia y Adrián Gimate Welsh, por México. Siguen las firmas de José Romera Castillo y de Paul Buissac, Patrick Imbert y Wladimir Krisinsky por España y Canadá y de Herman Parret, Iván Darrault y Per Aager Brandt por solidarios.

\section{EL ACTA DE BERKELEY (1994)}

Pasaron los años. Entre tanto la gente terminó sus doctorados, escribió libros, tuvo hijos, accedió a cátedras y cargos públicos, trabajó en el continente. Las asociaciones nacionales de semiótica siguieron con 
sus congresos. Durante mucho tiempo no nos volvimos a ver. Hasta que en junio de 1994 tuvo lugar en la universidad de Berkeley el V Congreso de la Asociación Internacional de Semiótica. Mientras pagaba el taxi que me había depositado en el muy español edificio del campus de la universidad, Rosa María Ravera acababa de llegar de Rosario. En la cola del self service apareció María Lucia Santaella Braga y Monica Rector charlando con Norma Tasca. Más allá, en la mesa de las inscripciones José María Paz Gago y Claudio Guerri intercambiaban opiniones sobre el sofocante verano californiano. A Adrián Gimate Welsh me lo encontré de casualidad - itantas eran las mesas redondas y la gente! - en la entrada de la conferencia de Paolo Fabbri, siguiendo seguramente una oscura fidelidad de oyentes. Con Teresa Espar, a la que no veía desde Rosario, compartimos una hamburguesa, mientras Rafael del Villar de Chile y Fernando Andacht de Uruguay se intercambiaban libros. Estábamos como siempre de un espléndido buen humor.

Corría el rumor de la candidatura de Finlandia para el próximo congreso internacional que debía ser ratificado en la votación de la Asamblea General. Lo que empezó a configurarse como la «Abominable banda de los ocho», se comprobó en el programa: más del cincuenta por ciento de los asistentes eran latinoamericanos, si nos organizábamos podríamos obtener la mayoría en la votación y dejar de pagar para ir a Europa a escuchar europeos -dicho sea sin ninguna predisposición contra los finlandeses - y hacer pagar a los europeos para escuchar a los latinoamericanos. En el fondo se trataba de un estricto problema de budget: sólo una gran pasión por la semiótica había hecho que durante una década, sin ningún presupuesto universitario, los latinoamericanos habíamos realizado travesías transoceánicas, vivido en las carísimas ciudades europeas, gracias al apoyo de nuestros amigos, viajado con kilos de libros de exceso de equipaje trotando por el viejo continente. Ahora queríamos quedarnos en casa, recibir al «otro» desde lo que habíamos hecho, mostrarnos como eramos.

En el bar «Berkeley's Serenade» nos citamos los latinos. Éramos muchísimos. Acordamos sostener la candidatura de México para el próximo Congreso Internacional y reflotar la Federación. Corrían vientos de fronda. Por fidelidad hacia mi maestro, fui hasta el hotel donde se alojaba Umberto Eco, flanqueda por Gimate Welsh y Guerri, para explicarle que nos íbamos a oponer por primera vez a la propuesta del Comité de la IAS y presentaríamos una candidatura autónoma. 
Eco pareció perplejo pero rápidamente dijo: «Irán a votación, y que gane la propuesta que tenga más votos». Durante toda la tarde preparamos el Acta de Berkeley, donde reflotábamos a la Federación, proponiendo México como sede para el Congreso Internacional en 1997 y San Pablo para organizar el Tercer Congreso Internacional Latinoamericano de Semiótica en 1996. En la noche del 16 de junio, entre cervezas y muchos mosquitos, realizamos nuestra primera asamblea luego de tantos años.

Cuando a la mañana siguiente llegamos a la sede de la asamblea de la Asociación Internacional de Semiótica, subí al podio junto con Gimate Welsh para leer nuestra propuesta: «Los abajo firmantes, miembros de la IASS/AIS y de las Asociaciones nacionales latinoamericanas queremos proponer formalmente la candidatura de México como sede para el Vl Congreso de la IASS/AIS. Las razones que nos impulsan a llevar adelante este apoyo tienen que ver con el deseo de una comunidad que se identifica con los valores profundos del saber. Fundamentamos nuestro pedido se basa en el creciente desarrollo de los estudios semióticos en América Latina y en la sostenida trayectoria mexicana en la producción y difusión de esta disciplina. La candidatura de México cuenta con el respaldo formal, económico e institucional de la Universidad Nacional Autónoma de México (doscientos cincuenta mil estudiantes), la Universidad Autónoma Metropolitana (ciento cincuenta mil estudiantes) y el Colegio de México, centro de excelencia en la investigación en Ciencias Sociales. Por otra parte, América Latina en general cuenta con cátedras de semiótica desde la década del setenta y con carreras de posgrado en semiótica en las principales universidades de Argentina, Brasil, Colombia, México, Perú, Uruguay y Venezuela. El dinamismo de sus investigadores se ha visto reflejado en la sistemática organización de congresos, coloquios, revistas y publicaciones a lo largo del continente y en la creación en 1987 de la Federación Latinoamericana de Semiótica. Creemos que nuestra propuesta apela al reconocimiento y a la dignidad de un país que en esta oportunidad representa un continente». Y seguían más de setenta firmas que habíamos recolectado.

Luego de un momento un poco tenso donde se enfrentaron las candidaturas, la votación nos fue favorable y estábamos tan felices que salimos a festejar atravesando, de atrás para adelante y de adelante para atrás varias veces, el puente del Golden Gate en caravana de automóviles alquilados, mientras en la radio escuchábamos a todo volumen «California Dreamming» y tocábamos corneta. 


\section{EL TERCER CONGRESO DE LA F.L.S (1996)}

Luego siguió el Tercer Congreso de la Federación Latinoamericana de Semiótica en agosto de 1996, organizado por María Lucia Santaella Braga de la Pontificia Universidad Católica de San Pablo, bajo el tema «Caos y Orden. Un abordaje semiótico». El III Congreso fue sencillamente gigantesco, como todo lo que organizan los brasileños. Sólo la edición del programa completo de actividades contaba doscientas veinte páginas 11. En la introducción, Santaella Braga escribía: «Durante muito tempo, caos e ordem foram vistos como opostos inconciliáveis. Negativamente considerado, caos era um mero sinónimo de desordem, fenómeno ou processo a ser evitado. Representava o desconhecido, o indomável, aquilo que escapava ao domínio da razzao e ao controle da lei. Há algunas décadas, entretanto, descobertas científicas passaram a apresentar o caos em interação indissolúvel com a ordem. Tanto a ordem pode estar oculta e passar despercebida no interior dos sistemas caóticos, quanto ela pode imprevisivlemente emergir do seio do caos graças a processos autoorganizativos. Como um fio invisível unindo todas as ondas do universo, a complementaridade entre o caos e a ordem se faz presente desde os fenómenos inorgánicos até as complexidades da vida humana. A semiótica, depois de terse ocupado principalmente com a elaboração dos sistemas semióticos, localizando-se, assim, no paradigma da ordem, começou a assumir o desafio dos novos modelos do caos na busca da ordem, sobretudo em processos evolutivos e criativos. De um ponto de vista transdisciplinar, o congresso de semiótica «Caos e Ordem», na PUCSP - 1996, dedicase aos novos desenvolvimientos na teoría do caos, estendendo-se por grandes áreas tais como a filosofia, as ciencias, as artes, a literatura e as mídias». Cuánto se había recorrido desde que usábamos la conjunción «y».

El congreso contó con ocho conferencias plenarias a cargo del gran poeta brasilero Haroldo de Campos, Solomon Marcus, Winfried Noth, Floyd Merrell, Thomas Sebeok y Roland Posner. Como la reunión era preparatoria del V Congreso Internacional de la IAS, que se celebraría en Guadalajara, luego de la histórica votación de Berkeley, el Comité

11 Caos e ordem. Uma abordagem semiótica. III Congresso Internacional Latinoamericano de Semiótica. IV Congresso Brasileiro de Semiótica. I Encontro de Semiótica Jurídica. Pontificia Universidad Católica de Sao Paulo, 31 de agosto-3 de setiembre, 1996. 
Ejecutivo de la Asociación Internacional estaba presente, así como la Secretaria Gloria Withalm y el responsable de la edición del boletín anual, Jeff Bernard, que era la primera vez que viajaban a Brasil. Las tres mesas plenarias -Caos y orden a la luz de la semiosis social, de la literatura, del arte y de la semiótica filósofica- así como las mesas temáticas que nuclearon a más de trescientos ponentes, dedicados a temas que nunca habíamos trabajado (como física, matématicas, nuevas tecnologías, discursos empresariales, previsibilidad e imprevisibilidad, imagen científica e imaginario artístico, religión, biología, dan$\mathrm{za}$, o ciencias cognitivas) marcaron un momento fuerte en la constitución no ya de una identidad latinoamericana, sino del grado de madurez e integración de sus representantes dentro de un escenario ya sin fronteras.

Siguiendo una tradición que nos trae buena suerte, en San Pablo redactamos el Acta de San Pablo, constituyendo a la primera comisión directiva electa con la presidencia del argentino Nicolás Rosa. El Acta fue firmada por colegas que no habían estado presentes en Berkeley, como el argentino Óscar Steimberg y por aquéllos para quienes era ya una tradición encontrarnos, como José Romera Castillo, José María Paz Gago o Araceli Herrero por España; Ana María Burdach y Rafael del Villar por Chile; Alejandro Russovich, Rosa María Brenca, Claudio Guerri, Rosa María Ravera, Roberto Marafioti, José Luis Fernández, Nicolás Rosa y yo por la Argentina; Fernando Andacht y Claudia González Costanzo por el Uruguay; Adrián Gimate Welsh y Katya Mandoki por México y José Enrique Finol por Venezuela. Norma Tasca estuvo presente por la Asociación Portuguesa de Semiótica. Releyendo el Acta original no me explico porqué no está firmada por ningún brasileño, visto que eran ellos los convocantes, pero seguramente en la confusión de la gente se les había escapado el ritual. Lo que quiero decir es algo muy simple: la Federación ha sido y es una aventura de todos, en el sentido más amplio del colectivo de identificación «todos».

Finalmente durante el V Congreso de la Asociación Internacional de Semiótica en setiembre de 1997, en Guadalajara (México), con el lema «La semiótica entre naturaleza y cultura» y una gran serpiente Quetzacoatl en forma de «S», escribimos el Acta de Guadalajara, con casi ochenta firmas, solicitando a la Asociación Internacional la incorporación del español como lengua oficial junto al francés y el inglés. Jorge Lozano, con el que había compartido mi formación en la universidad de Bolonia, con Umberto Eco, se incorporó por primera vez a 
nuestro grupo, defendiendo la posición española. La discusión en la Asamblea General de la IAS - tras repartirse un boletín informativo en cuatro lenguas (inglés, francés, español y portugués) de las actividades semióticas realizadas en Latinoamérica, España y Portugal, bajo la inspiración de José Romera Castillo - fue memorable, por estar muy enraizada la problématica de la lengua como vehículo de identidad cultural. José Ángel Fernández Roca, de la Asociación Gallega de Semiótica y Secretario General del próximo congreso de la FLS, al salir de la asamblea, decía que hacía tiempo que no se discutía algo con tanta pasión. ¿Existen los semiólogos iberoamericanos? Tal vez sean (o no) una ficción latinoamericana. 